1

Multibody Syst Dyn manuscript No.

(will be inserted by the editor)

The final publication is available at Springer via http://dx.doi.org/10.1007/s11044-011-9260-0

\title{
Parameter identification in dynamic systems using the homotopy optimization approach
}

\author{
Chandrika P. Vyasarayani - Thomas Uchida · \\ Ashwin Carvalho • John McPhee
}

Received: date / Accepted: date

\begin{abstract}
Identifying the parameters in a mathematical model governed by a system of ordinary differential equations is considered in this work. It is assumed that only partial state measurement is available from experiments, and that the parameters appear nonlinearly in the system equations. The problem of parameter identification is often posed as an optimization problem, and when deterministic methods are used for optimization, one often converges to a local minimum rather than the global minimum. To mitigate the problem of converging to local minima, a new approach is proposed for applying the homotopy technique to the problem of parameter identification. Several examples are used to demonstrate the effectiveness of the homotopy method for obtaining global minima, thereby successfully identifying the system parameters.
\end{abstract}

Keywords Parameter estimation · Global optimization · Homotopy · Reduced-order modelling $\cdot$ Partial state measurement

\section{Introduction}

The problem of identifying the parameters in a mathematical model governed by ordinary differential equations (ODEs), given a set of experimental measurements, is encountered in many fields of physics, chemistry, biology, and engineering [8]. The problem of parameter identification can be posed as an optimization problem $[17,18]$, where the arguments of the global minimum of the objective function are the identified parameters. If the parameters appear linearly in the system equations and full state measurement is available from experimental data, a large class of methods is available for both off-line and on-line parameter identification $[17,18]$. In most practical engineering problems, however, it is not possible to

C.P. Vyasarayani $(\bowtie) \cdot$ T. Uchida $\cdot$ J. McPhee

Department of Systems Design Engineering, University of Waterloo

200 University Avenue West, Waterloo, Ontario, N2L 3G1, Canada

E-mail: cpvyasar@uwaterloo.ca, tkuchida@uwaterloo.ca, mcphee@real.uwaterloo.ca

A. Carvalho

Indian Institute of Technology Bombay, Powai, Mumbai, 400076, India

E-mail: ashwincarvalho@iitb.ac.in 
obtain measurements for all states, and the parameters often appear nonlinearly in the equations of motion. Off-line identification algorithms are used when the main goal is to develop a mathematical model for system simulation; on-line identification algorithms are more popular in adaptive control applications [15]. The optimization problems are usually solved using deterministic methods, which require the solution of differential equations at each optimization step. The solution of these ODEs can be obtained using initial-value methods [10, 26], shooting methods [1], or collocation methods [2]. When deterministic approaches like the steepest descent [22], Gauss-Newton [22], and Levenberg-Marquardt [20] algorithms are used in the optimization procedure, it is not uncommon to converge to a local minimum rather than the global minimum [7]. Stochastic methods, such as simulated annealing [23] and genetic algorithms [9], can be used to find global minima, but these methods typically require a large number of iterations to converge and, thus, are time-consuming, especially for parameter identification problems where the equations of motion are integrated at every optimization step $[10,11,26]$. An obvious question that arises is whether there exist any nonstochastic algorithms that can find global minima. Although one can never be assured that a deterministic algorithm will be able to find the global minimum in every situation, there are approaches derived from homotopy methods that can find global minima in situations where other deterministic methods cannot.

Homotopy [27] is a powerful technique that is used in several areas of mathematics, including optimization [5,12] and nonlinear root finding [4]. In homotopy methods, the objective function to be minimized is modified by adding another function whose optimum is known, herein referred to as the known function, and a morphing parameter is used to transform the modified function into the original objective function. A series of optimizations is performed while slowly varying the morphing parameter until the modified function is transformed back into the original objective function [5]. Applying homotopy to algebraic optimization problems is straightforward, but its application to the parameter identification problem is not, since the objective function is, itself, dependent on the solution of differential equations. Homotopy was successfully applied to ARMAX models [13,14] for the identification of linear parameters. In the work of Abarbanel et al. [1], the authors have coupled the mathematical model to the experimental data for identifying parameters from a chaotic time series for first-order systems, which is related to the homotopy method. The application of homotopy to the general nonlinear parameter identification problem has not been studied in the literature. In this work, we present a methodology to apply homotopy to the problem of parameter identification. We show several examples where the classical deterministic methods fail to find the global minimum while the homotopy method successfully minimizes the objective function.

\section{Problem Statement}

The dynamic equations of the physical system for which the parameters must be identified are assumed to be of the following form:

$$
\begin{aligned}
& \dot{\boldsymbol{x}}_{1}=\boldsymbol{x}_{2} \\
& \dot{\boldsymbol{x}}_{2}=\boldsymbol{f}\left(\boldsymbol{x}_{1}, \boldsymbol{x}_{2}, \boldsymbol{p}, t\right)
\end{aligned}
$$

For mechanical systems, $\boldsymbol{x}_{1}(t)=\left[y_{1}(t), y_{2}(t), \ldots, y_{n}(t)\right]^{\mathrm{T}}$ are the independent coordinates (displacements) and $\boldsymbol{p}$ is a column vector containing the parameters to be identified. The system is assumed to be nonlinear, and the parameters may also appear nonlinearly in 
equations (1). Experimental data $\boldsymbol{x}_{1 e}(t)=\left[y_{1 e}(t), y_{2 e}(t), \ldots, y_{n e}(t)\right]^{\mathrm{T}}$ of all the displacements are assumed to be available over time $T$; it is assumed that the velocities are not measured. Note that it is possible to identify the system parameters with only a few components of $\boldsymbol{x}_{1 e}(t)$ since, in coupled systems, each component of $\boldsymbol{x}_{1 e}(t)$ contains information from all the parameters due to the coupling between the system equations. The unknown initial conditions of the states for which measurements are not available can be treated as unknown parameters. In order to simplify the optimization procedure, experiments can be performed starting from rest, such that the velocity initial conditions are zero. The goal is to identify the parameters in the mathematical model such that the solution of the differential equations (1) closely matches the experimental data. To identify the parameters, we minimize the integral of the squared difference between the experimental and simulated states:

$$
V(\boldsymbol{p})=\frac{1}{2} \sum_{i=1}^{n}\left\{\int_{0}^{T}\left(y_{i e}(t)-y_{i}(t, \boldsymbol{p})\right)^{2} d t\right\}
$$

Note that a discrete summation can be used in place of the integral in this equation. Given the initial estimates of the parameters, we can minimize equation (2) iteratively using the Gauss-Newton method [22] as follows:

$$
\boldsymbol{p}^{r+1}=\boldsymbol{p}^{r}+\sigma \boldsymbol{d}^{r}
$$

where $\sigma$ is the step size and $\boldsymbol{d}^{r}$ is the search direction, which can be obtained from the following relation [26]:

$$
\boldsymbol{H}\left(\boldsymbol{p}^{r}\right) \boldsymbol{d}^{r}=-\boldsymbol{G}^{\mathrm{T}}\left(\boldsymbol{p}^{r}\right)
$$

In equation (4), $\boldsymbol{G}$ and $\boldsymbol{H}$ are the gradient and the approximate Hessian of the objective function, where the second-order sensitivities have been neglected in the latter:

$$
\begin{aligned}
& \boldsymbol{G}(\boldsymbol{p})=\frac{\partial V}{\partial \boldsymbol{p}}=-\sum_{i=1}^{n}\left\{\int_{0}^{T}\left(y_{i e}(t)-y_{i}(t, \boldsymbol{p})\right) \frac{\partial y_{i}}{\partial \boldsymbol{p}} d t\right\} \\
& \boldsymbol{H}(\boldsymbol{p})=\frac{\partial^{2} V}{\partial \boldsymbol{p}^{2}} \approx \sum_{i=1}^{n}\left\{\int_{0}^{T} \frac{\partial y_{i}}{\partial \boldsymbol{p}} \frac{\partial y_{i}}{\partial \boldsymbol{p}} d t\right\}
\end{aligned}
$$

The sensitivity data $\frac{\partial y_{i}}{\partial \boldsymbol{p}}=\left[\frac{\partial y_{i}}{\partial p_{1}}, \frac{\partial y_{i}}{\partial p_{2}}, \ldots, \frac{\partial y_{i}}{\partial p_{m}}\right]$ can be obtained by solving the sensitivity differential equations, which can be derived by the direct differentiation of equations (1) with respect to the individual parameters:

$$
\begin{aligned}
\frac{\partial \dot{\boldsymbol{x}}_{1}}{\partial p_{j}}= & \frac{\partial \boldsymbol{x}_{2}}{\partial p_{j}} \\
\frac{\partial \dot{\boldsymbol{x}}_{2}}{\partial p_{j}}= & \frac{\partial \boldsymbol{f}\left(\boldsymbol{x}_{1}, \boldsymbol{x}_{2}, \boldsymbol{p}, t\right)}{\partial p_{j}}+\frac{\partial \boldsymbol{f}\left(\boldsymbol{x}_{1}, \boldsymbol{x}_{2}, \boldsymbol{p}, t\right)}{\partial \boldsymbol{x}_{1}} \frac{\partial \boldsymbol{x}_{1}}{\partial p_{j}}+\frac{\partial \boldsymbol{f}\left(\boldsymbol{x}_{1}, \boldsymbol{x}_{2}, \boldsymbol{p}, t\right)}{\partial \boldsymbol{x}_{2}} \frac{\partial \boldsymbol{x}_{2}}{\partial p_{j}} \\
& j=1,2, \ldots, m
\end{aligned}
$$

We will briefly explain how homotopy is applied to a simple algebraic minimization problem. Let $\mathcal{F}(\boldsymbol{p})$ be the objective function. We are interested in finding parameters $\boldsymbol{p}^{*}$ at which $\mathcal{F}$ has a global minimum. If we start from an arbitrary point $\boldsymbol{p}^{0}$, and if the function 
has multiple local minima, it is likely that the optimization procedure will converge to a local minimum. In the homotopy method, we first construct the following function:

$$
\mathcal{H}(\boldsymbol{p}, \lambda)=(1-\lambda) \mathcal{F}(\boldsymbol{p})+\lambda \mathcal{G}(\boldsymbol{p})
$$

where $\mathcal{G}(\boldsymbol{p})$, referred to as the known function, is a chosen function that is convex in the unknown parameters, and for which the arguments of its global minimum are known. We now begin the process by choosing $\lambda_{0}=1$ and minimizing $\mathcal{H}(\boldsymbol{p}, 1)=\mathcal{G}(\boldsymbol{p})$. In this first stage, the minimum of $\mathcal{H}(\boldsymbol{p}, 1)$ is simply the minimum of $\mathcal{G}(\boldsymbol{p})$, which is known. Once the minimum has been found, $\lambda$ is decreased by a small amount $\delta \lambda$ and $\mathcal{H}\left(\boldsymbol{p}, \lambda_{1}\right)$ is minimized, where $\lambda_{\ell}=1-\ell \delta \lambda$, using the converged result from the previous stage as the initial guess for $\boldsymbol{p}$. This process is continued until $\lambda=0$ and the objective function has been morphed back into $\mathcal{F}(\boldsymbol{p})$. Provided we are always finding the minimum of $\mathcal{H}(\boldsymbol{p}, \lambda)$ with an initial guess that is close to its global minimum, it is more likely that we will find the global minimum of the function $\mathcal{F}(\boldsymbol{p})$. A variant of this method has been successfully applied to complex optimization problems involving protein structures [6] and to finding the equilibrium configuration of an elastica [28]. Note that the choice of known function $\mathcal{G}(\boldsymbol{p})$ is nontrivial. In general, the homotopy method is only capable of finding local minima; however, if the nature of $\mathcal{F}(\boldsymbol{p})$ is known, it may be possible to construct the homotopy transformation in a way that increases the chance of finding the global minimum. It is for this reason that the optimization problem for parameter identification is generally more challenging than it is for purely algebraic problems. In particular, the shape of $\mathcal{F}(\boldsymbol{p})=V(\boldsymbol{p})$ is unknown in parameter identification problems, since it is dependent on the solution of differential equations (1), thereby making the selection of a suitable known function $\mathcal{G}(\boldsymbol{p})$ very difficult. In contrast to algebraic problems, however, where the minimum value of the objective function is unknown, the minimum value of the objective function in parameter identification problems is zero-provided the mathematical model is known exactly. Since the mathematical model is not precisely known in general, the final error may not be exactly zero; however, it is expected to be small. We use this knowledge in developing our homotopy transformation.

We now discuss how the homotopy method can be applied to the problem of parameter identification. To modify the objective function, the experimental data is coupled to the mathematical model as follows:

$$
\begin{aligned}
& \dot{\boldsymbol{x}}_{1}=\boldsymbol{x}_{2}+\lambda K_{1}\left(\boldsymbol{x}_{1 e}-\boldsymbol{x}_{1}\right) \\
& \dot{\boldsymbol{x}}_{2}=\boldsymbol{f}\left(\boldsymbol{x}_{1}, \boldsymbol{x}_{2}, \boldsymbol{p}, t\right)+\lambda K_{2}\left(\boldsymbol{x}_{1 e}-\boldsymbol{x}_{1}\right)
\end{aligned}
$$

Initially, when $\lambda=1$, the coupling term acts as a high-gain observer $[3,16]$, and if sufficiently high values of $K_{i}$ are used, the experimental data and simulated response will synchronize. Note that $\lambda$ is introduced to the traditional definition of a high-gain observer so as to construct the homotopy transformation. Also note that the sensitivity equations (7) must be modified to account for the added coupling term. For very large $K_{i}$, the objective function becomes a flat surface with a very small magnitude, and the experimental data $\boldsymbol{x}_{1 e}$ and simulated response $\boldsymbol{x}_{1}$ will closely match no matter what parameters are used. For the purposes of parameter identification, we choose the lowest values of $K_{i}$ such that the experimental data and simulated response synchronize to within a desired tolerance $\varepsilon$ when the initial parameter estimates are used. We now decrease $\lambda$ by a small amount $\delta \lambda$ and minimize the objective function (2), treating equation (9) as the mathematical model. The parameter estimates are refined so as to reduce the error to $\beta \varepsilon$, where $0<\beta<1$. We then decrease $\lambda$ further to $1-2 \delta \lambda$; since the parameter guesses have been refined, the observer gain can be 
reduced without increasing the error beyond $\varepsilon$. At each stage in this process, we use the converged result from the previous stage as the initial guess for $\boldsymbol{p}$. This process is repeated until $\lambda=0$, and equation (9) has morphed back into equation (1). In summary, the homotopy optimization approach follows the path of minimal error as the observer gain is decreased. We ensure that the error is close to zero (no greater than $\varepsilon$ ) at each value of the observer gain, with the hope that the refined parameter guesses at the final stage are sufficiently close to the global optimum of the original problem. The process of applying the homotopy method to the problem of parameter identification is summarized in Algorithm 1.

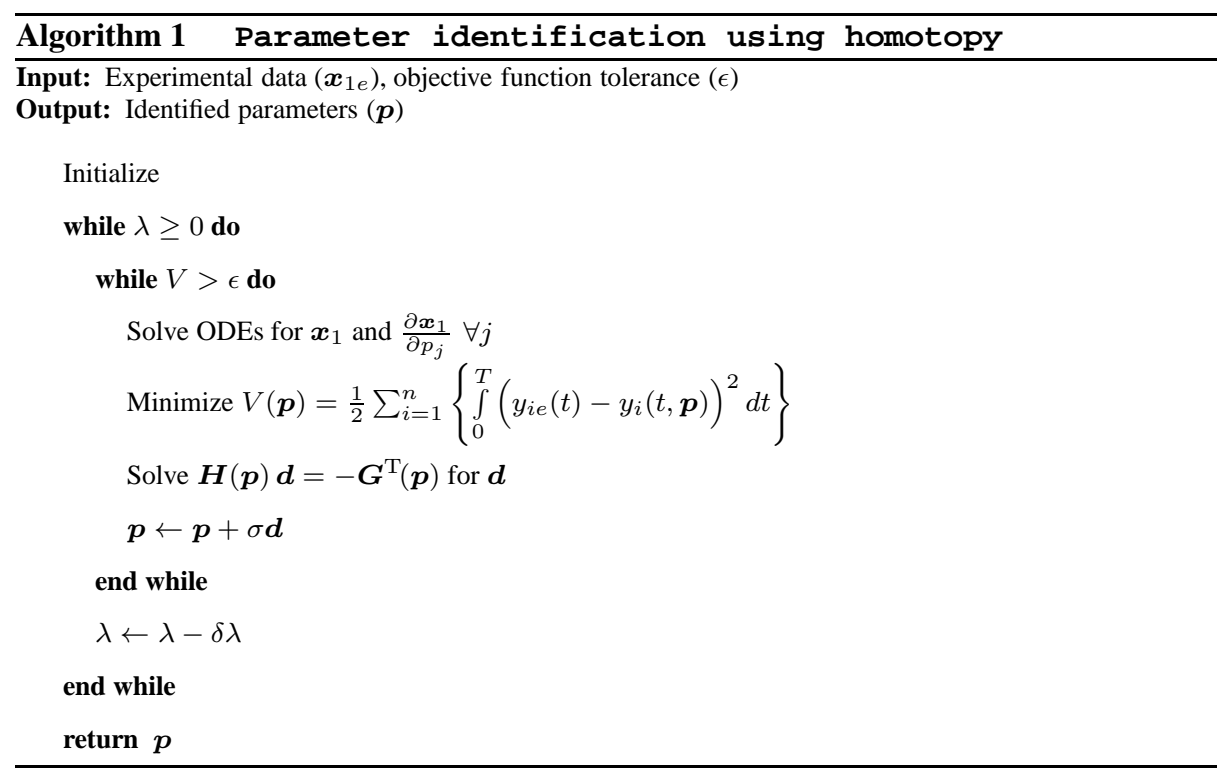

\section{Numerical Examples}

In this section, we present numerical examples in which the homotopy method has been used to successfully identify the parameters in mechanical systems.

\subsection{Linear parameters}

Let us begin with the identification of parameters for a simple pendulum. We describe this example in detail to further explain the idea of the homotopy method. The equations of motion for a simple pendulum are given in state-space form as follows:

$$
\begin{aligned}
& \dot{y}_{1}=y_{2} \\
& \dot{y}_{2}=-p \sin \left(y_{1}\right)
\end{aligned}
$$

with initial conditions $y_{1}(0)=\pi / 6$ and $y_{2}(0)=0$. The solution of this system with $p=10$ for time $t \in[0,50]$ is used as experimental data $y_{1 e}$. The goal is to determine $p$ from the 
experimental data. We define the following minimization problem:

$$
p^{*}=\min _{\arg } \int_{0}^{50}\left(y_{1 e}-y_{1}(p)\right)^{2} d t
$$

A direct search has been used to identify the shape of the objective function, as shown in Figure 1. It can be seen that the objective function has multiple local minima, and any

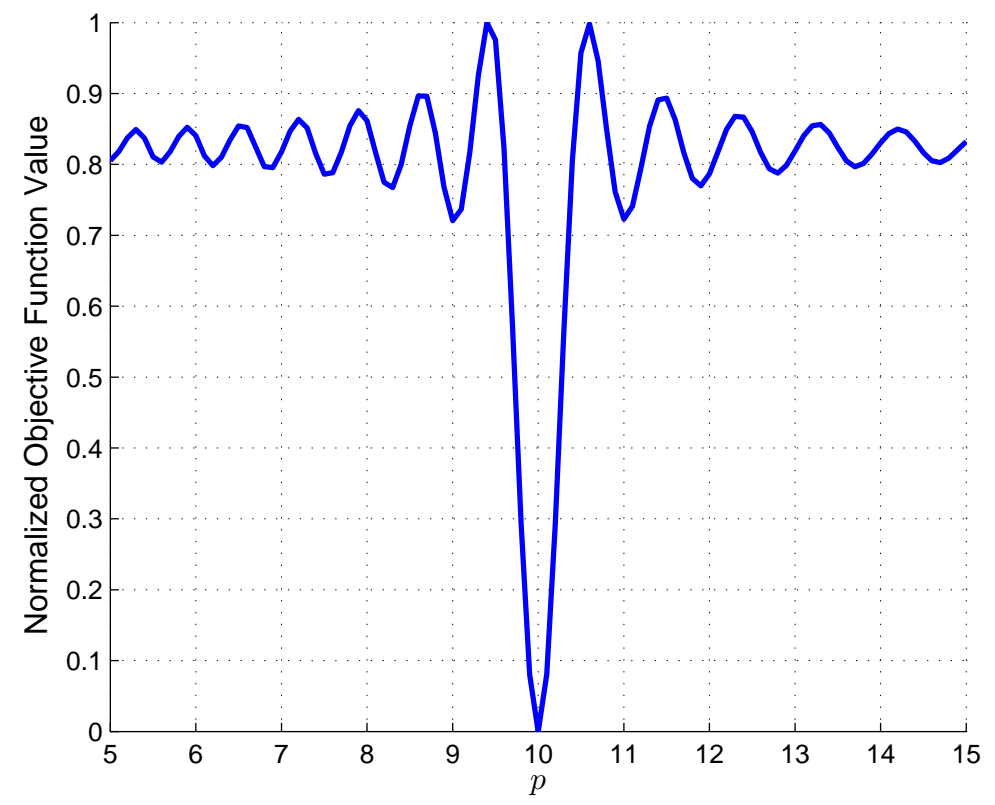

Fig. 1 Normalized objective function for simple pendulum

classical deterministic optimization method will fail to converge to the global minimum unless we choose our initial guess for $p$ to be quite close to 10. To apply the homotopy method, we modify the equations of motion as follows:

$$
\begin{aligned}
& \dot{y}_{1}=y_{2}+\lambda K_{1}\left(y_{1 e}-y_{1}\right) \\
& \dot{y}_{2}=-p \sin \left(y_{1}\right)+\lambda K_{2}\left(y_{1 e}-y_{1}\right)
\end{aligned}
$$

The sensitivity equations are now given as follows:

$$
\begin{aligned}
& \frac{\partial \dot{y}_{1}}{\partial p}=\frac{\partial y_{2}}{\partial p}-\lambda K_{1} \frac{\partial y_{1}}{\partial p} \\
& \frac{\partial \dot{y}_{2}}{\partial p}=-\sin \left(y_{1}\right)-p \cos \left(y_{1}\right) \frac{\partial y_{1}}{\partial p}-\lambda K_{2} \frac{\partial y_{1}}{\partial p}
\end{aligned}
$$

Figure 2, again obtained using a direct search, illustrates the shape of the objective function for different values of $\lambda$ and $p$, using coupling parameters $K_{1}=K_{2}=10$. As can be seen, the modified objective function is convex when $\lambda=1$; as we decrease $\lambda$ to 0 , the modified 
objective function slowly morphs into the original objective function shown in Figure 1. The line joining the minimum of the modified function for different values of $\lambda$ is also shown. We have implemented the homotopy method for identifying $p$ as illustrated in Algorithm 1, with $\epsilon=0.001$ and $\delta \lambda=0.2$. Using the homotopy method with an initial guess of $p^{0}=15$, we converge to the global minimum at $p=10$ since we always remain close to the global minimum of the modified objective function. Without using homotopy, again starting with an initial guess of $p^{0}=15$, the parameter converges to a local minimum at $p=14.6755$.

\subsection{Nonlinear parameters}

Consider the following system, which has been studied in [3]:

$$
\ddot{u}+0.1 \dot{u}+\tan ^{-1}(k u)=\sin \left(\frac{t}{2}+\pi \psi\right)
$$

The system is nonlinear in both $k$ and $\psi$. We use $k=1$ and $\psi=0.5$ for generating experimental data, and then attempt to identify these parameters using initial guesses $k^{0}=4$ and $\psi^{0}=2$. The identification procedure was first performed without using homotopy, the results of which are shown in Figure 3(a). Clearly, the optimization procedure (GaussNewton) has converged to a local minimum where $k=4.5850$ and $\psi=1.9620$. The homotopy method was then applied using the same initial guesses for $k$ and $\psi$. In this example, 
we use coupling parameters $K_{1}=K_{2}=10$, step size $\delta \lambda=0.2$, and objective function tolerance $\epsilon=0.01$. As shown in Figure 3(b), the global minimum was found and both parameters were correctly identified using the homotopy method (we obtain $k=1.0001$ and $\psi=2.5000$ ). Note that the estimate for $\psi$ produces an equivalent response as does $\psi=0.5$, since $\sin \left(\frac{t}{2}+\pi(\psi+2)\right)=\sin \left(\frac{t}{2}+\pi \psi\right)$.
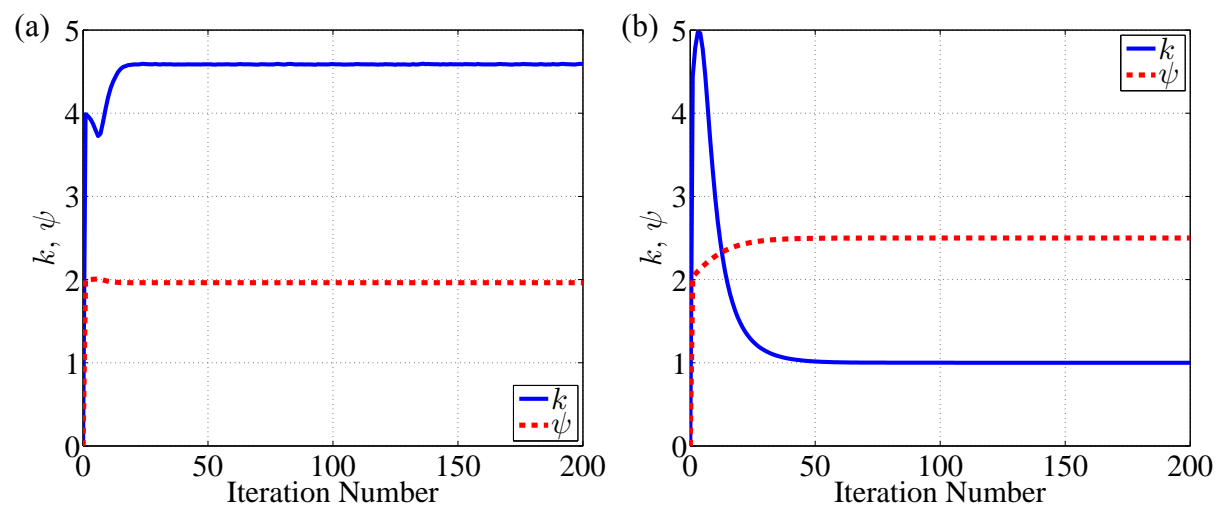

Fig. 3 Parameter estimates for equation (14) obtained (a) without homotopy, and (b) with homotopy

\subsection{Noisy experimental data}

Consider the following two-degree-of-freedom (DOF) system [3]:

$$
\begin{aligned}
\ddot{u}+0.3 \dot{u}+u+C_{1} u^{3}+C_{2} v & =0 \\
\ddot{v}+0.3 \dot{v}+u+C_{3} v^{3}+\cos (t) & =0
\end{aligned}
$$

where $C_{1}=1, C_{2}=2$, and $C_{3}=3$ are used for generating the experimental data for $u$ and $v$, to which we add $5 \%$ white noise. In this example, we assume that the initial conditions for all states are known. Taking as initial guesses $C_{1}^{0}=C_{2}^{0}=C_{3}^{0}=10$ and without using homotopy, the optimization procedure converges to parameters that are different from the experimental values (we obtain $C_{1}=13.3840, C_{2}=7.9444$, and $C_{3}=9.3622$, as shown in Figure 4(a)). Although we do not expect to achieve exact convergence due to the noise in the experimental data, these parameter values are significantly different than the actual values. Using the homotopy method with $K_{i}=10 \forall i$ and $\delta \lambda=0.2$, we obtain parameter estimates $C_{1}=0.9998, C_{2}=2.0008$, and $C_{3}=3.0034$, which are very close to the experimental values (see Figure 4(b)). Note that only the experimental data for $u$ is coupled to the equations of motion; it is assumed that the data for $v$ is not measured. This coupling strategy confirms that it is not necessary to measure all the coordinates of a multiple-DOF system in order to identify all the system parameters. The experimental data and the results from the simulated system using the identified parameters are in good agreement, as shown in Figure 5. 

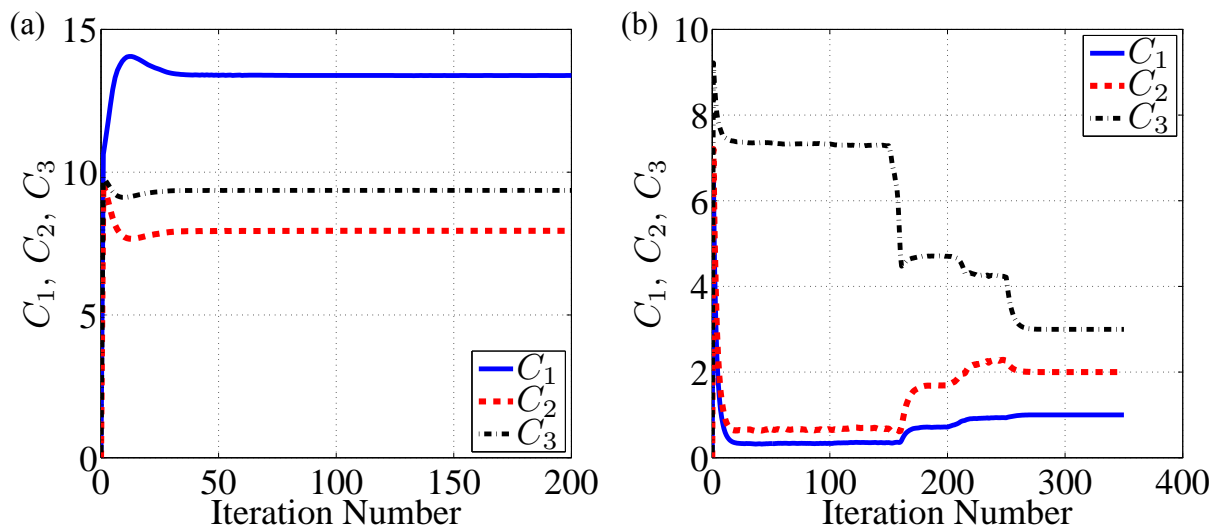

Fig. 4 Parameter estimates for equation (15) obtained (a) without homotopy, and (b) with homotopy

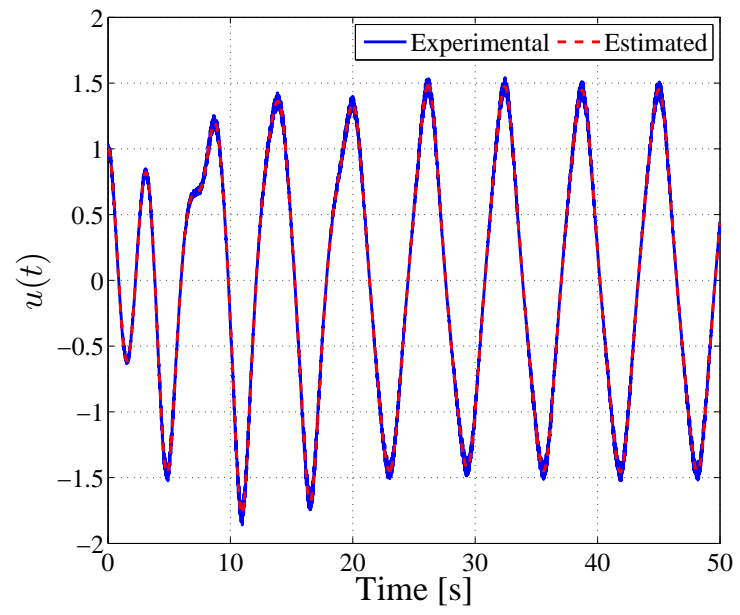

Fig. 5 Experimental data and simulated response for equation (15) using identified parameters

\subsection{Reduced-order modelling}

In this final example, we consider the problem of identifying parameters in a multibody system given experimental data generated using a more complex model. In particular, a 14-DOF vehicle model with a fully independent suspension, shown in Figure 6, is used to generate the experimental data. This topology is recommended by Sayers [24] for simulating the handling and braking behaviour of a vehicle, and has been adopted by several commercial software packages. The position and orientation of the vehicle chassis $\left(m_{c}\right)$ together comprise 6 DOF. Four lumped masses $\left(m_{s}\right)$, each representing one-quarter of the suspension components, are connected to the chassis by prismatic joints in parallel with springs $(k)$ and dampers $(c)$, which represent the suspension compliance and together add 4 DOF. Each wheel is connected to its corresponding lumped mass with a horizontally-oriented revolute joint that allows the wheel to spin, collectively accounting for the final $4 \mathrm{DOF}$; torques $(\tau(t))$ can be applied at these joints to accelerate the vehicle. The Pacejka2002 Magic Formula tire 


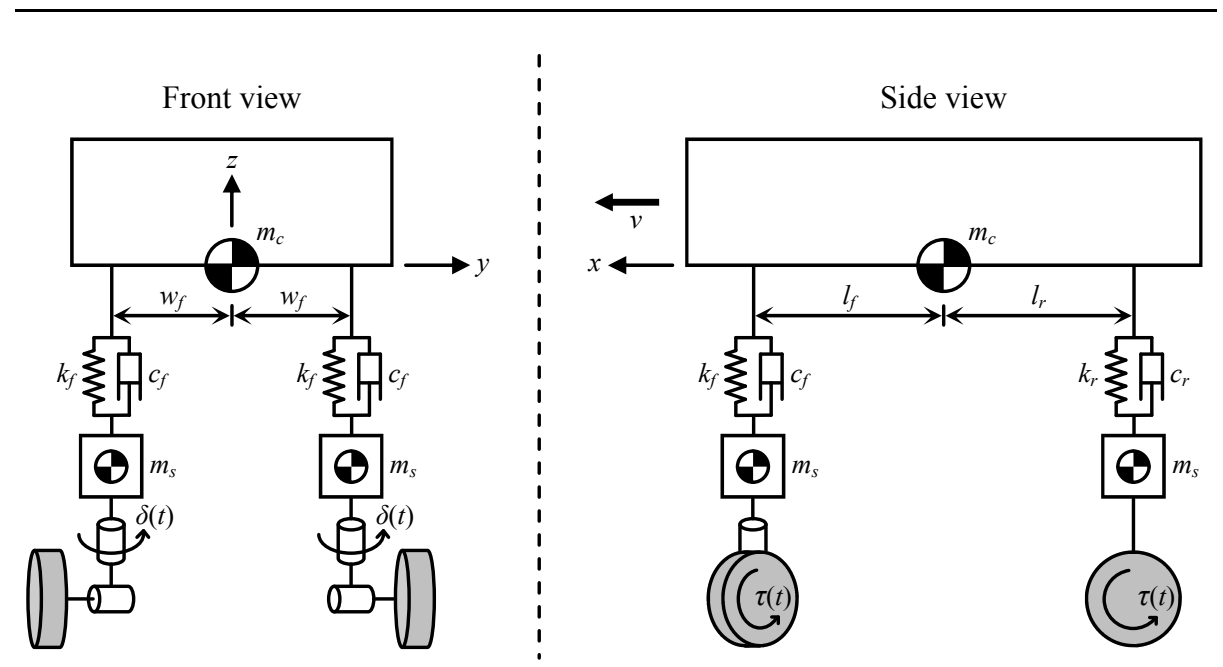

Fig. 6 14-DOF vehicle model used for generating experimental data

model [21] is used to model the tire dynamics. The vertically-oriented revolute joints on the front wheels $(\delta(t))$ are used to steer the vehicle on a prescribed trajectory, so do not add any DOF to the system. The system parameters are obtained from [25], some of which are shown in Table 1.

Table 1 System parameters for 14-DOF vehicle model

\begin{tabular}{lll}
\hline Parameter & & Value \\
\hline \multirow{2}{*}{ Mass } & Chassis $\left(m_{c}\right)$ & $2077 \mathrm{~kg}$ \\
& Quarter of suspension $\left(m_{s}\right)$ & $10 \mathrm{~kg}$ \\
Stiffness & Front suspension $\left(k_{f}\right)$ & $48.30 \mathrm{kN} / \mathrm{m}$ \\
& Rear suspension $\left(k_{r}\right)$ & $30.52 \mathrm{kN} / \mathrm{m}$ \\
Damping & Front suspension $\left(c_{f}\right)$ & $3.08 \mathrm{kN}-\mathrm{s} / \mathrm{m}$ \\
& Rear suspension $\left(c_{r}\right)$ & $2.33 \mathrm{kN}-\mathrm{s} / \mathrm{m}$ \\
& Front width $\left(w_{f}\right)$ & $0.760 \mathrm{~m}$ \\
Dimensions & Rear width $\left(w_{r}\right)$ & $0.795 \mathrm{~m}$ \\
& Front length $\left(l_{f}\right)$ & $1.353 \mathrm{~m}$ \\
& Rear length $\left(l_{r}\right)$ & $1.487 \mathrm{~m}$ \\
\hline
\end{tabular}

Experimental data generated from the 14-DOF model with Pacejka tires is used to identify parameters in the planar bicycle model shown in Figure 7. The planar bicycle model is often used for the simulation of vehicle dynamics and for on-board stability controllers. This simple model has only 3 DOF: the position of the chassis in the $x$-direction $\left(y_{1}\right)$, the position of the chassis in the $y$-direction $\left(y_{2}\right)$, and the orientation of the chassis in the plane $\left(y_{3}\right)$. The mass of the chassis is assumed to be $m_{b}=m_{c}+4 m_{s}=2117 \mathrm{~kg}$, and the lengths are assumed to be $d_{f}=l_{f}=1.353 \mathrm{~m}$ and $d_{r}=l_{r}=1.487 \mathrm{~m}$. The yaw inertia of the chassis is also defined to match that of the 14-DOF model. A simple 4-parameter tire model [19] 


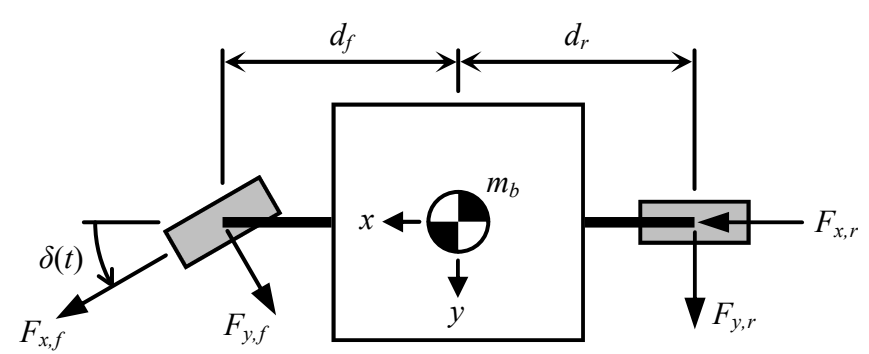

Fig. 7 Planar vehicle model for which parameters are sought

is used in place of the complex 117-parameter Pacejka model used in the 14-DOF system:

$$
\begin{aligned}
s_{b} & =1-\frac{v_{x, w}}{R \omega} \\
F_{x, w} & =A_{\text {long }}\left(1-e^{-B_{\text {long }} s_{b}}\right) F_{z} \\
\alpha_{b} & =\arctan \left(\frac{v_{y, w}}{v_{x, w}}\right) \\
F_{y, w} & =A_{\text {lat }}\left(1-e^{-B_{\text {lat }} \alpha_{b}}\right) F_{z}
\end{aligned}
$$

where $s_{b}$ is the longitudinal slip, $\alpha_{b}$ is the lateral slip angle, $v_{x, w}$ and $v_{y, w}$ are the longitudinal and lateral components of the wheel velocity in the wheel reference frame, $R$ is the tire radius, and $\omega$ is the rotational speed of the wheel. Forces $F_{x, w}$ and $F_{y, w}$ are applied directly to the wheel centers. The tire radius $(R)$ is assumed to be equal to the unloaded radius used in the Pacejka model, and the vertical tire force $\left(F_{z}\right)$ is simply assumed to be half the total static load in the 14-DOF model. We wish to find values for the tire parameters $\left(A_{\text {long }}, B_{\text {long }}, A_{\text {lat }}\right.$, and $B_{\text {lat }}$ ) such that the simulated response of the 3-DOF planar model matches the experimental data obtained from the 14-DOF model as closely as possible. Note that the planar model has no suspension and can neither pitch nor roll.

To generate the experimental data, we must first define the required inputs. In order to adequately capture both the longitudinal and lateral dynamics, we first accelerate the vehicle from $4 \mathrm{~m} / \mathrm{s}$ to $16 \mathrm{~m} / \mathrm{s}$ by applying positive torque to each wheel, then perform a lanechange maneuver, and finally slow the vehicle to $6 \mathrm{~m} / \mathrm{s}$ by applying negative torque to each wheel. The steer angle and wheel torque inputs are shown in Figure 8. Using these inputs, the 14-DOF vehicle model is simulated for 22 seconds and the state vector is stored every millisecond. For the purpose of parameter identification, we assume that only five states are known: the position of the chassis along the $x$ - and $y$-axes, the orientation of the chassis about the $z$-axis, and the average rotational speeds of the two front and two rear wheels. Note that the wheel speeds of the 14-DOF model are required in order to calculate the tire forces applied to the 3-DOF model (equations (16) to (19)). We use the following objective function:

$$
V=\sum_{i=1}^{3}\left\{w_{i} \int_{0}^{22}\left(y_{i e}(t)-y_{i}\left(t, A_{\text {long }}, B_{\text {long }}, A_{\text {lat }}, B_{\text {lat }}\right)\right)^{2} d t\right\}
$$

where weights $w_{1}=0.001, w_{2}=0.01$, and $w_{3}=1$ are chosen to scale the longitudinal position, lateral position, and orientation errors to the same orders of magnitude. We obtain 
12

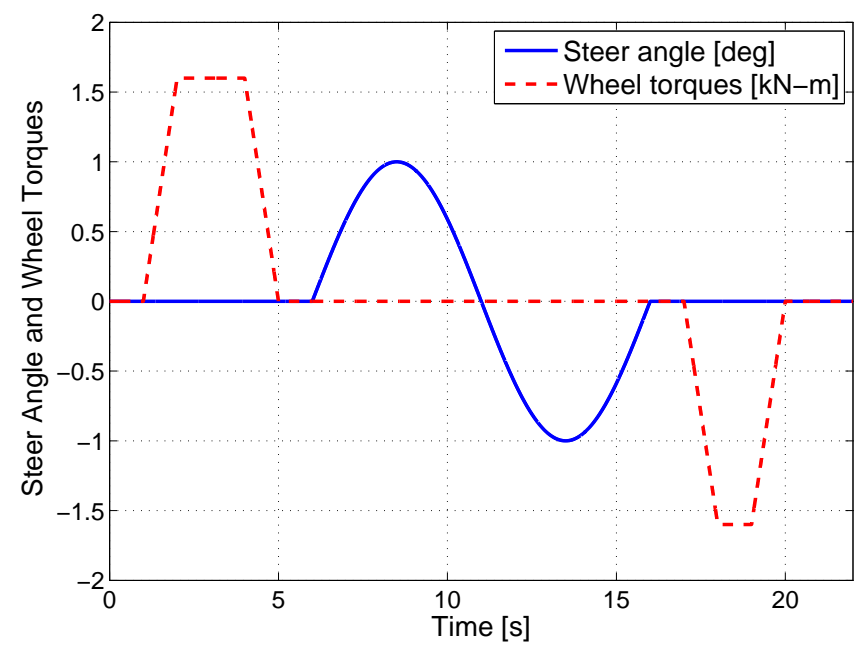

Fig. 8 Inputs used to generate experimental data with the 14-DOF model

rough initial guesses $A_{l o n g}=B_{l o n g}=100$ and $A_{l a t}=B_{l a t}=1$ by hand, which corresponds to an objective function value of $1.804 \times 10^{-2}$ and produces the simulation results shown in Figure 9.
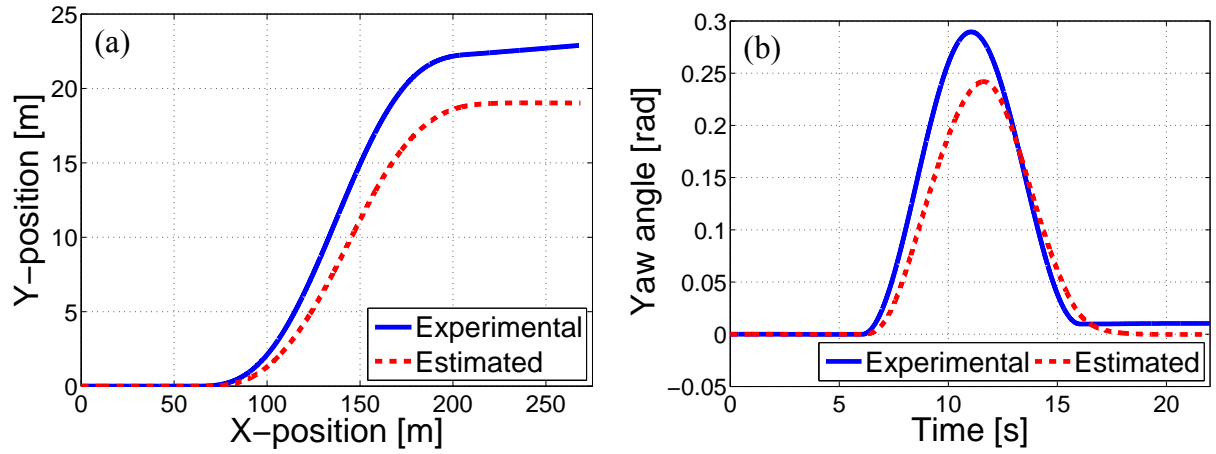

Fig. 9 Simulation results for (a) trajectory and (b) yaw angle, obtained using initial parameter guesses

We perform the optimization procedure using the homotopy method described above, with $K_{i}=0.5 \forall i, \delta \lambda=0.1$, and $\epsilon=10^{-4}$. Convergence is achieved after a total of 12 iterations, with a final objective function value of $V_{f}=3.3 \times 10^{-5}$, as shown in Figure 10 . The identified parameters are $A_{\text {long }}=99.97, B_{\text {long }}=99.97, A_{\text {lat }}=3.05$, and $B_{\text {lat }}=$ 3.06; the corresponding simulation results are shown in Figure 11. 


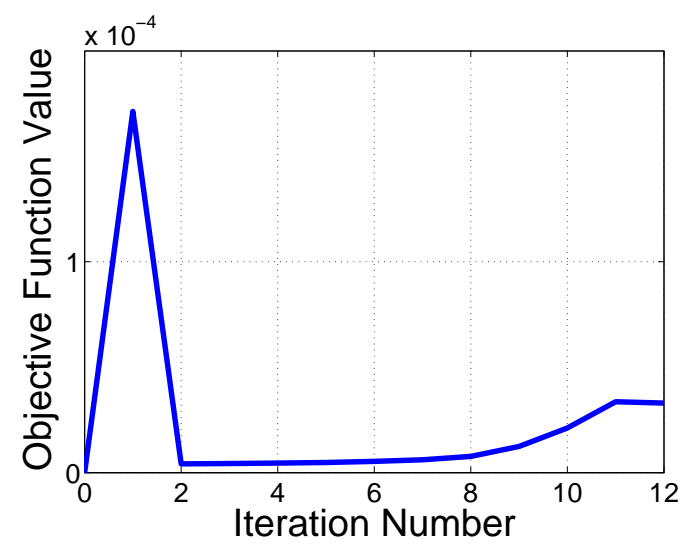

Fig. 10 Convergence of the objective function (normalized by the number of data points)
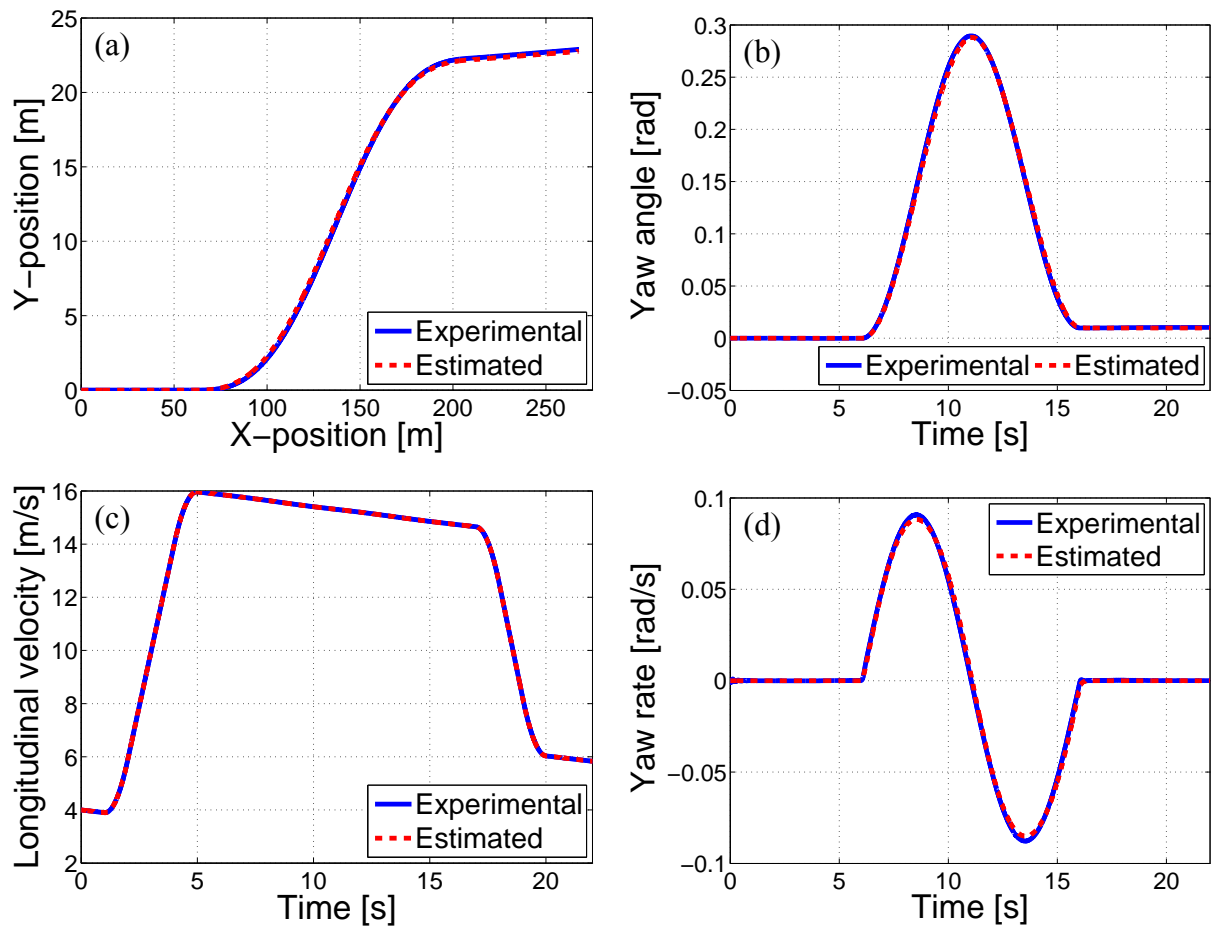

Fig. 11 Simulation results for (a) trajectory, (b) yaw angle, (c) longitudinal velocity, and (d) yaw rate, obtained using identified parameters 


\section{Conclusions}

In this work, we have presented a new methodology for applying the homotopy optimization technique to the parameter identification problem. We have considered the general problem of parameter identification for nonlinear parameters with partial state measurement corrupted by measurement noise. The proposed homotopy method can successfully find global minima given a wide range of initial parameter guesses. The effectiveness of the proposed technique for parameter identification has been demonstrated by several example problems. The authors are currently investigating the use of the homotopy method for recursive parameter identification in on-line applications, and for systems governed by differential-algebraic equations.

Acknowledgements The financial support provided to the first author by the Ontario Centres of Excellence, to the second author by the Natural Sciences and Engineering Research Council of Canada (NSERC), to the third author by the MITACS Globalink Program, and to the fourth author by the NSERC/Toyota/Maplesoft Industrial Research Chair program is gratefully acknowledged. The first author also wishes to acknowledge Professor Kingshook Bhattacharyya, IIT Kharagpur and Professor Baris Fidan, University of Waterloo for several interesting discussions during the course of this study.

\section{References}

1. Abarbanel, H.D.I., Creveling, D.R., Farsian, R., Kostuk, M.: Dynamical state and parameter estimation. SIAM J. Appl. Dyn. Syst. 8, 1341-1381 (2009)

2. Brewer, D., Barenco, M., Callard, R., Hubank, M., Stark, J.: Fitting ordinary differential equations to short time course data. Philos. Trans. R. Soc. Lond., Ser. A 366, 519-544 (2008)

3. Chatterjee, A., Cusumano, J.P.: Asymptotic parameter estimation via implicit averaging on a nonlinear extended system. J. Dyn. Syst. Meas. Control 125, 11-18 (2003)

4. Chow, S.N., Mallet-Paret, J., Yorke, J.A.: A homotopy method for locating all zeros of a system of polynomials. Funct. Differ. Equs. Approx. Fixed Points 730, 77-88 (1979)

5. Dunlavy, D.M., O'Leary, D.P.: Homotopy optimization methods for global optimization. Sandia National Laboratories, Report SAND2005-7495 (2005)

6. Dunlavy, D.M., O'Leary, D.P., Klimov, D., Thirumalai, D.: HOPE: A homotopy optimization method for protein structure prediction. J. Comput. Biol. 12, 1275-1288 (2005)

7. Eberhard, P., Schiehlen, W., Bestle, D.: Some advantages of stochastic methods in multicriteria optimization of multibody systems. Arch. Appl. Mech. 69, 543-554 (1999)

8. Gershenfeld, N.: The nature of mathematical modeling. Cambridge University Press (1999)

9. Goldberg, D.E.: Genetic Algorithms in Search, Optimization and Machine Learning. Addison-Wesley (1989)

10. Gordon, T.J., Hopkins, R.: Parametric identification of multibody models for crash victim simulation. Multibody Syst. Dyn. 1, 85-112 (1997)

11. He, Y., McPhee, J.: Application of optimisation algorithms and multibody dynamics to ground vehicle suspension design. Int. J. Heavy Veh. Syst. 14, 158-192 (2007)

12. Hillermeier, C.: Generalized homotopy approach to multiobjective optimization. J. Optim. Theory Appl. 110, 557-583 (2001)

13. Hu, J., Hirasawa, K.: A homotopy approach to identification of ARMAX systems. Trans. Inst. Electr. Eng. Jpn. 119, 206-211 (1999)

14. Hu, J., Hirasawa, K., Kumamaru, K.: A homotopy approach to improving PEM identification of ARMAX models. Automatica 37, 1323-1334 (2001)

15. Ioannou, P., Fidan, B.: Adaptive control tutorial. Society for Industrial and Applied Mathematics (2006)

16. Khalil, H.K.: High-gain observers in nonlinear feedback control, Lecture Notes in Control and Information Sciences: New Directions in Nonlinear Observer Design, vol. 244, pp. 249-268. Springer (1999)

17. Ljung, L.: System identification: Theory for the user. Prentice-Hall (1987)

18. Ljung, L., Söderström, T.: Theory and practice of recursive identification. MIT Press (1983)

19. Metz, L.D.: Dynamics of four-wheel-steer off-highway vehicles. SAE Paper No. 930765 (1960)

20. Moré, J.J.: The Levenberg-Marquardt algorithm: Implementation and theory. Numer. Analysis 630, 105-116 (1977) 
21. Pacejka, H.B.: Tire and Vehicle Dynamics. SAE International (2002)

22. Rao, S.S.: Optimization: Theory and Applications. John Wiley \& Sons (1983)

23. Romeijn, H.E., Smith, R.L.: Simulated annealing for constrained global optimization. J. Global Optim. 5, 101-126 (1994)

24. Sayers, M.W., Han, D.: Generic multibody vehicle model for simulating handling and braking. Veh Syst. Dyn. 25, 599-613 (1996)

25. Schmitke, C., Morency, K., McPhee, J.: Using graph theory and symbolic computing to generate efficient models for multibody vehicle dynamics. IMechE J. Multi-body Dyn. 222, 339-352 (2008)

26. Serban, R., Freeman, J.S.: Identification and identifiability of unknown parameters in multibody dynamic systems. Multibody Syst. Dyn. 5, 335-350 (2001)

27. Watson, L.T., Haftka, R.T.: Modern homotopy methods in optimization. Comput. Methods Appl. Mech. Eng. 74, 289-305 (1989)

28. Watson, L.T., Wang, C.Y.: A homotopy method applied to elastica problems. Int. J. Solids Struct. 17, 29-37 (1981) 